\title{
Optic nerve sheath fenestration for vision preservation in idiopathic intracranial hypertension
}

\author{
Madhu R. Agarwal, M.D., ${ }^{1}$ AND Ji Hye YoO $^{2}$ \\ ${ }^{1}$ Department of Ophthalmology, ${ }^{2}$ Loma Linda University School of Medicine, Loma Linda, California
}

\begin{abstract}
$\checkmark$ Idiopathic intracranial hypertension, also known as pseudotumor cerebri, is a condition of increased intracranial pressure without the presence of mass lesions and with normal cerebrospinal fluid composition. Patients may experience papilledema and vision loss. Optic nerve sheath fenestration (ONSF) is one method of stabilizing visual function and decreasing optic nerve edema. The authors report on 10 patients who underwent bilateral ONSF and in whom visual function was stable or improved postoperatively. The results obtained in these cases suggest that ONSF plays a role in visual preservation in idiopathic intracranial hypertension patients in the acute setting.
\end{abstract} (DOI: 10.3171/FOC-07/11/E7)

\section{KEY WORDS • idiopathic intracranial hypertension • optic nerve sheath fenestration • pseudotumor cerebri}

I DIOPATHIC intracranial hypertension, also referred to as pseudotumor cerebri, is a condition of elevated ICP in which hydrocephalus and vascular or mass lesion are absent and normal CSF composition is present. It occurs most frequently in young, obese women, most commonly in their third and fourth decades. ${ }^{16}$ Ophthalmological consequences of increased ICP include transient blurred vision, diplopia from nonlocalizing abducens nerve palsy, papilledema, and sudden or progressive loss of visual acuity or visual field. Although between 25 and $50 \%$ of patients experience some loss of vision, this visual impairment may not be readily appreciated by the patient. ${ }^{4,17}$ Timely diagnosis of visual disturbances caused by $\mathrm{IIH}$ is critical to allow the implementation of medical or surgical interventions.

Ideally, the treatment of IIH would both preserve visual function and treat the symptoms of the elevated ICP, including headache. Nonsurgical management includes weight loss and the use of medications such as carbonic anhydrase inhibitors and loop diuretics. When medical therapy fails or visual function declines, surgical therapies should be considered. The two main surgical modalities are LP shunt placement and ONSF.

Both LP shunt therapy and ONSF have been shown to stabilize or improve visual function in cases of elevated ICP. ${ }^{1,14}$ This retrospective review of 10 consecutive cases of bilateral optic nerve sheath fenestrations performed by one surgeon (M.R.A.) over a 1-year period (2006) demonstrates the utility of ONSF in cases of acute and progressive visual loss.

\footnotetext{
Abbreviations used in this paper: $\mathrm{CSF}=$ cerebrosponal fluid; $\mathrm{ICP}=$ intracranial pressure; IIH = idiopathic intracranial hypertension; LP = lumboperitoneal; ONSF = optic nerve sheath fenestration.
}

\section{Case Reports}

Informed consent for ONSF was obtained from all 10 patients after extensive neuroophthalmological evaluations revealed decreases in visual acuity or visual fields. Idiopathic intracranial hypertension was diagnosed using appropriate imaging and CSF evaluations. ${ }^{7}$ All surgeries were performed bilaterally via a medial transconjunctival approach (Fig. 1). ${ }^{8}$ Postoperative changes in visual acuity and visual fields (as determined by automated Humphrey central threshold testing, $30^{\circ}$ on each side of midline) were noted. To confirm that visual improvement was secondary to ONSF, the follow-up period was intentionally limited to the postoperative period before any further medical or surgical interventions were undertaken. Table 1 summarizes the pre- and postoperative visual function noted in the 10 cases.

Case 1. This 17-year-old obese boy was diagnosed with $\mathrm{IIH}$ and started on a course of acetazolamide. One week later the patient was referred for neuroophthalmological examination because he had experienced 3 days of severe vision loss, with light perception in the right eye, 20/70 vision in the left eye, and severe bilateral disc edema. Because of severe nausea, the patient declined to undergo visual field testing. Bilateral ONSF was performed that night. On postoperative Day 1, right- and left-sided vision was $20 / 200$ and 20/50, respectively. Two weeks later, vision was unchanged, and visual field testing showed superior restriction in the right eye and normal fields in the left. Two months later, with the patient was still receiving the same acetazolamide regimen, and visual acuity was 20/80 in the right eye and 20/30 in the left; visual fields were unchanged, but the optic nerves were flat bilaterally.

Case 2. This 31-year-old woman diagnosed with IIH and 


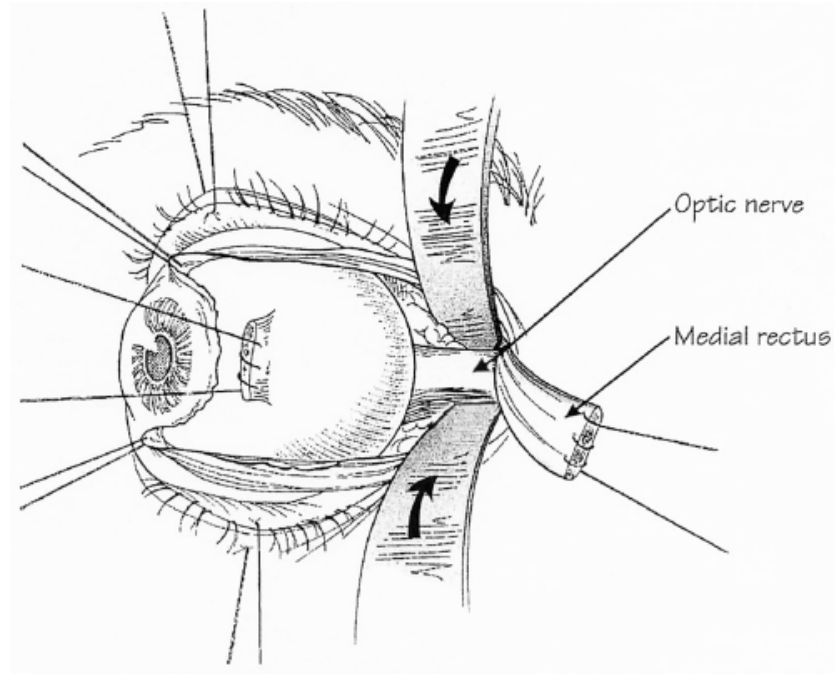

FIG. 1. Diagram depicting medial transconjunctival orbitotomy involving takedown of the medial rectus to expose optic nerve. Curved black arrows show the optic nerve. Reproduced with permission from Glaser JS (ed): Neuro-Ophthalmology, ed 3. Philadelphia: Lippincott-Williams \& Wilkins, 1999.

taking acetazolamide presented with 20/20 vision bilaterally, severe bilateral disc edema, and worsening visual field loss in the superior aspect of both eyes. One week after undergoing bilateral ONSF, she had full visual fields and decreased disc swelling.

Case 3. This 7-year-old boy with IIH and taking no medication presented with progressive vision loss. On hospital admission, his vision was limited to finger counting with the right eye and 20/80 vision in the left eye. The optic nerves were pale with some bilateral edema. Bilateral optic nerve sheath fenestration was performed 2 days later, resulting in visual acuity of 20/400 in the right eye and 20/80 in the left within 1 week.

Case 4. This 24-year-old man had a several-month history of severe headaches for which he was being observed. He was eventually diagnosed with IIH after a sudden catastrophic bilateral vision loss to light perception. Although he used acetazolamide, no improvement in vision or disc edema was noted. Bilateral OSNF was performed on the day of referral. One week postoperatively, vision was 20/60 in the right eye and 20/50 in the left, with a dramatic decrease in disc edema (Fig. 2) and 8-prism-diopter esotropia. His visual field remained severely limited. Four weeks after surgery, vision was 20/25 bilaterally with straight eyes, with approximately $15^{\circ}$ of central visual field bilaterally.

Case 5. This 39-year-old woman presented with IIH and a 2-week history of worsening vision. Presenting visual acuity was 20/80 in the right eye and 20/100 in the left, with inferior visual field loss bilaterally. The following day, after bilateral ONSF, vision was 20/50 bilaterally.

Case 6. This 28-year-old man was diagnosed with IIH. History included multiple shunt revisions and a 2-month history of progressive vision loss. During this time, he was closely observed but refused further intervention as his vision rapidly declined from $20 / 20$ to $20 / 400$ bilaterally with poor visual fields and significant disc edema. The
TABLE 1

Visual function before and after bilateral ONSF*

\begin{tabular}{cll}
\hline \hline $\begin{array}{c}\text { Case } \\
\text { No. }\end{array}$ & \multicolumn{1}{c}{ Preop Deficit } & \multicolumn{1}{c}{ Postop Result } \\
\hline 1 & light only OD, 20/70 OS & 20/200 OD, 20/50 OS \\
2 & decrease in superior visual field OU & full fields OU \\
3 & finger counting OD, 20/80 OS & $20 / 400$ OD, 20/80 OS \\
4 & light only OU & $20 / 60$ OD, 20/50 OS \\
5 & $20 / 80$ OD, 20/100 OS & $20 / 50$ OU \\
6 & $20 / 400$ OD, 20/400 OS & $20 / 400$ OU \\
7 & 20/80 OD, 20/30 OS & $20 / 40$ OD, 20/30 OS \\
8 & $20 / 100$ OD, 20/25 OS & $20 / 30$ OD, 20/25 OS \\
9 & $20 / 80$ OD, 20/40 OS & $20 / 60$ OD, 20/25 OS \\
10 & light only OD, finger counting & light only OD, finger \\
& 1 ft OS & counting 6 ft OS \\
\hline
\end{tabular}

* $\mathrm{OD}=$ right eye, $\mathrm{OS}=$ left eye; $\mathrm{OU}=$ both eyes.

patient ultimately agreed to undergo bilateral ONSF and surgery was performed the following day. Two weeks postoperatively, his vision remained at 20/400 bilaterally, but the discs were no longer edematous.

Case 7. This 25-year-old man, who was receiving acetazolamide treatment, presented with $\mathrm{IIH}$, severe headaches, disc edema, 20/80 vision in the right eye, and 20/30 vision in the left. Examination of the right visual fields showed almost complete loss and the left field showed superior loss. Bilateral ONSF was performed the following day. Headaches persisted. On postoperative Day 1, vision was 20/40 in the right eye and 20/30 in the left.

Case 8. This 52-year-old man with IIH reported decreasing vision while receiving acetazolamide. Presenting acuity was $20 / 100$ in the right eye and 20/25 in the left, with a right afferent pupillary defect, optic nerve edema, atrophy bilaterally, severe visual field loss in the right eye, and temporal loss in the left. One week after bilateral ONSF, visual acuity was 20/30 in the right eye and 20/25 in the left, with some inferior field loss in the right and full visual field in the left eye.

Case 9. This 43-year-old woman with chronic IIH presented with bilateral disc edema; she was receiving acetazolamide and complained of worsening vision. Visual acuity was $20 / 80$ on the right side and 20/40 on the left, with bilateral disc swelling, severe visual field loss in the right eye, and temporal loss in the left eye. On Day 3 after bilateral ONSF, right- and left-sided visual acuity was 20/60 and 20/25, without afferent pupillary defect.

Case 10. This 21-year-old man with IIH and a history of multiple shunts and ONSF bilaterally complained of severe left-sided loss of vision. Visual acuity at bedside was light perception in the right eye and finger counting at 1 foot in the left, with optic atrophy bilaterally and combined atrophy and edema in the left eye. Lumbar puncture revealed elevated ICP, and a bilateral ONSF was performed to attempt to salvage vision. On postoperative Day 1, vision was light perception in the right eye and finger counting at 6 feet in the left, with no change in disc appearance.

\section{Discussion}

Idiopathic intracranial hypertension may lead to visual loss due to the following postulated mechanisms: 1) axon- 

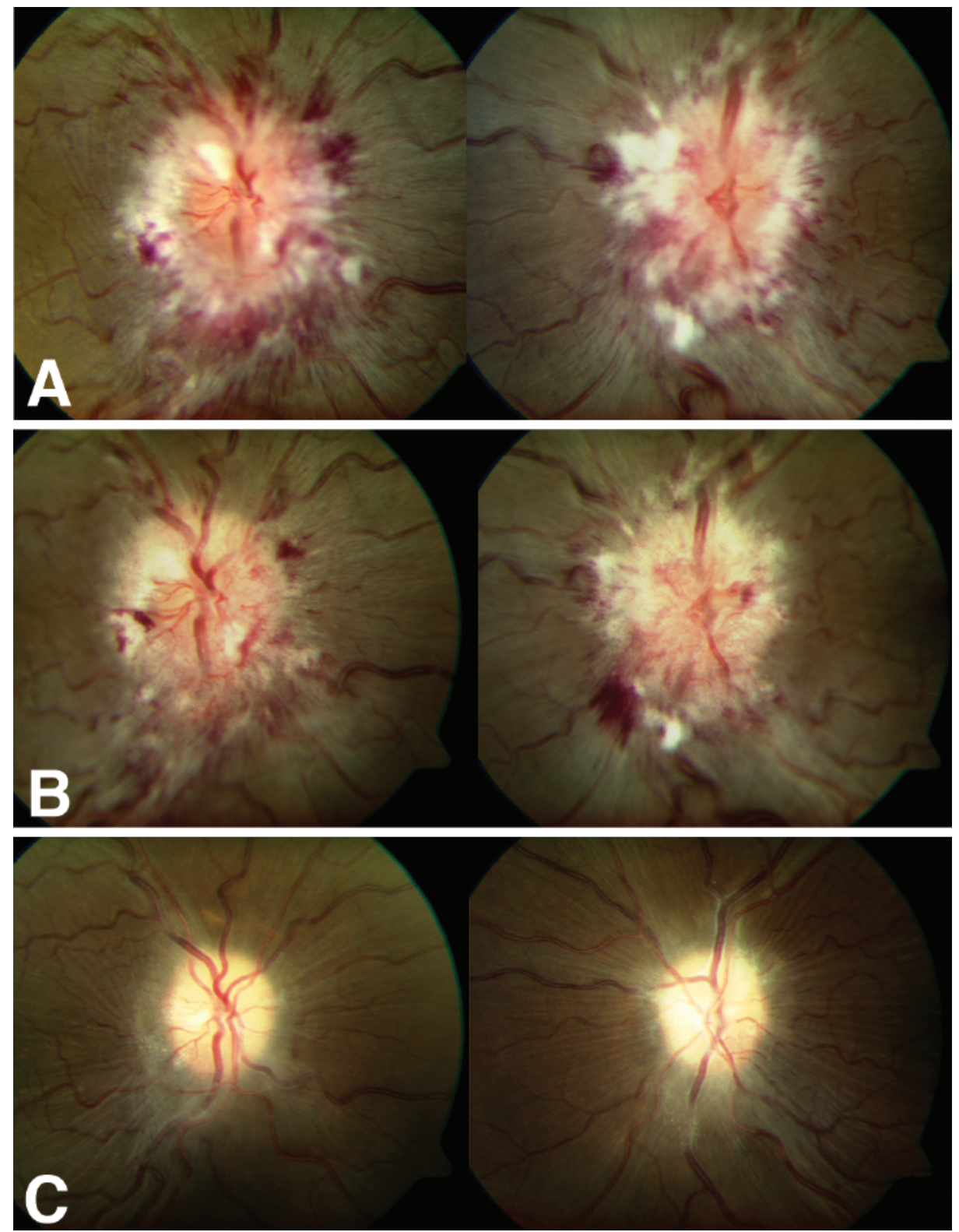

FIG. 2. Case 4. Ophthalmological examinations. A: Severe nerve fiber layer infarcts and hemorrhages, as well as swelling in the right and left optic nerves, respectively, just prior to ONSF. B: Day 7 after ONSF, some resolution of papilledema can be observed. Vision during this period improved from light perception bilaterally to 20/60 in the right eye and 20/50 in the left. C: Right and left optic nerves 4 weeks after ONSF, illustrating further reduction of swelling.

al stasis within the nerve and resulting ischemia; 2) orbital venous stasis and hypertension; and 3) traction on optic pathways resulting from dilated ventricles. ${ }^{3}$ All of these mechanisms may play a role. Some investigators have suggested that ONSF may alleviate the CSF pressure on the nerve, improving axoplasmic flow. ${ }^{12}$ Mild, acute disc edema may therefore respond better to ONSF than severe, long-standing edema. Furthermore, prolonged, increased ICP may stimulate arachnoid proliferation in the orbital subarachnoid space, generating further pressure on the nerve. ${ }^{13}$ The results in our Case 6 support this finding because this is the only patient in the series in whom visual acuity did not improve, but who had chronic, severe papil- ledema. It is difficult to make further observations about the relationship between the duration of symptoms and surgical results because the duration was highly variable among all 10 cases.

It is also possible that ONSF may act as a "mini-shunt," because it allows CSF to leak into the orbit. Data provided by Tsai and colleagues ${ }^{15}$ have demonstrated that ONSF may result in a fibrotic bleb, filtering CSF over time. It has also been shown that unilateral ONSF may decrease papilledema bilaterally, demonstrating its shuntlike effects. ${ }^{10}$ However, because our patients required interventions including LP shunt placement and medication, we do not believe that ONSF acts as a sole modality of treatment. Many 
factors play a role in patients' visual outcome, including compliance, changes in medications, and surgical interventions. Separate studies by Burgett and coworkers ${ }^{2}$ and Eggenberger and colleagues ${ }^{6}$ have shown that LP shunt therapy in IIH has significantly improved or stabilized visual function in these patients. 2,6 However, several patients (Cases 6 and 10) in our case series continued to experience visual loss despite having undergone previous LP shunt procedures or medications. This finding may be explained by the highly variable degree of communication that exists between the orbital and intracranial subarachnoid spaces. ${ }^{9}$ Shunt therapy may not necessarily decrease pressure on the optic nerve. In our cases, ONSF was able to directly decrease optic nerve swelling and improve or stabilize visual fields and visual acuity.

Many authors have noted prolonged visual improvement after ONSF. ${ }^{13,16}$ However, long-term IIH data can be confounded by the multiple medical and surgical treatments that patients may undergo. Indeed, several of our patients required other surgeries or medications to alleviate symptoms such as recalcitrant headache (Cases 5 and 7). To mitigate the effects of these confounding variables, the data shown here were limited to those obtained in the acute postoperative period.

One complication seen in this series was the 8-prismdiopter esotropia that occurred in Case 4, which was caused by takedown of the medial rectus muscle and its resuturing into position after the fenestration of the optic nerve. The esotropia seen in Case 4 resolved over the ensuing 4 weeks. The medial rectus muscle may be avoided completely by using a superomedial orbitotomy approach. The authors of other studies have noted a complication rate of $40 \%$, including vascular compromise or occlusions, ocular motility problems, and pupillary dysfunction. ${ }^{11}$ Most complications were transient and caused no permanent sequelae. However, this complication rate may not have occurred in the present case series because of the small number of cases. Previous studies have also included cases of disc edema with various causes, including ischemic optic neuropathy or compressive optic neuropathy, which may behave differently than IIH. Many other reported cases were reoperations, which may result in further complications. Finally, because of the infrequent need for ONSF in many academic centers, the surgeon or surgical team may be less familiar with this procedure, thus contributing to a higher complication rate.

It may be that neither ONSF nor shunt procedures alone can address both headache management and vision preservation. Rather, treatment must be tailored to the needs of each patient, requiring a multidisciplinary approach. There is little question that the treatment of patients with $\mathrm{IIH}$ can be extremely difficult. However, with the fourfold increase in obesity, the primary risk factor for $\mathrm{IIH}$, between 1988 and 2002, improved treatments and algorithms must be investigated. ${ }^{5}$ More data should be obtained from a ran- domized controlled clinical trial in which investigators directly compare the utility of medications, ONSF, and LP shunt therapy.

\section{References}

1. Binder DK, Horton JC, Lawton MT, McDermott MW: Idiopathic intracranial hypertension. Neurosurgery 54:538-552, 2004

2. Burgett RA, Purvin VA, Kawasaki A: Lumboperitoneal shunting for pseudotumor cerebi. Neurology 49:734-739, 1997

3. Chou SY, Digre KB: Neuro-ophthalmic complications of raised intracranial pressure, hypdrocephalus, and shunt malfunction. Neurosurg Clin N Am 10:587-608, 1999

4. Corbett JJ, Savino PJ, Thompson HS, Kansu T, Schatz NJ, Orr LS, et al: Visual loss in pseudotumor cerebri. Follow-up of 57 patients from five to 41 years and a profile of 14 patients with permanent severe visual loss. Arch Neurol 39:461-474, 1982

5. Curry WT Jr, Butler WE, Barker FG II: Rapidly rising incidence of cerebrospinal fluid shunting procedures for idiopathic intracranial hypertension in the United States, 1988-2002. Neurosurgery 57:97-108, 2005

6. Eggenberger ER, Miller NR, Vitale S: Lumboperitoneal shunt for the treatment of pseudotumor cerebri. Neurology 46:1524-1530, 1996

7. Friedman DI, Jacobson DM: Diagnostic criteria for idiopathic intracranial hypertension. Neurology 59:1492-1495, 2002

8. Galbraith JE, Sullivan JH: Decompression of the perioptic meninges for relief of papilledema. Am J Ophthalmol 76:687-692, 1973

9. Hayreh SS: Pathogenesis of oedema of the optic disc. Doc Ophthalmol 24:289-411, 1968

10. Keltner J: Optic nerve sheath decompression. How does it work? Has its time come? Arch Ophthalmol 106:1365-1369, 1988

11. Plotnik JL, Kosmorsky GS: Operative complications of optic nerve sheath decompression. Ophthalmology 100:683-690, 1993

12. Seiff SR, Shah L: A model for the mechanism of optic nerve sheath fenestration. Arch Ophthalmol 108:1326-1329, 1990

13. Sergott RC, Savino PJ, Bosley TM: Modified optic nerve sheath decompression provides long-term visual improvement for pseudotumor cerebri. Arch Ophthalmol 106:1384-1390, 1988

14. Spoor TC, McHenry JG: Long-term effectiveness of optic nerve sheath decompression for pseudotumor cerebri. Arch Ophthalmol 111:632-635, 1993

15. Tsai JC, Petrovich MS, Sadun AA: Histopathological and ultrastructural examination of optic nerve sheath decompression. Br J Ophthalmol 79:182-185, 1995

16. Wall M, George D: Idiopathic intracranial hypertension. A prospective study of 50 patients. Brain 114:155-180, 1991

17. Wall M, Hart WM Jr, Burde RM: Visual field defects in idiopathic intracranial hypertension (pseudotumor cerebri). Am J Ophthalmol 96:654-669, 1983

Manuscript submitted July 10, 2007.

Accepted September 5, 2007.

Address correspondence to: Madhu R. Agarwal, M.D., Department of Ophthalmology, Loma Linda University Faculty Medical Offices, 11370 Anderson Street, Suite 1800, Loma Linda, California 92354. email: magarwal@ahs.llumc.edu. 